\title{
Avaliação da atividade tóxica em Artemia salina e Biomphalaria glabrata de extratos de quatro espécies do gênero Eleocharis (Cyperaceae)
}

\author{
A.L.T.G. Ruiz ${ }^{1,2 *}$, E.G. Magalhães ${ }^{1}$, A.F. Magalhães ${ }^{1}$, A.D. Faria ${ }^{3}$, M.C.E. Amaral ${ }^{3}$, \\ D.R. Serrano ${ }^{3}$, E.M. Zanotti-Magalhães ${ }^{3}$, L.A. Magalhães $^{3}$. \\ ${ }^{1}$ Departamento de Química Orgânica do Instituto de Química, UNICAMP, C.P. 6154, 13084-971, \\ Campinas, SP, Brasil \\ ${ }^{2}$ Departamento de Botânica do Instituto de Biologia, UNICAMP, C.P 6109, 13084-971, Campinas, SP, Brasil \\ ${ }^{3}$ Departamento de Parasitologia do Instituto de Biologia, UNICAMP, C.P 6109,13084-971, Campinas, SP, Brasil
}

\begin{abstract}
RESUMO: O gênero Eleocharis R. Br. compreende cerca de 200 espécies, ocorrendo em ambientes úmidos tais como brejos e margens de rios e lagos. Procurando novos agentes moluscicidas, os extratos de Eleocharis acutangula (Roxb.) Schult., Eleocharis interstincta (Vahl) Roem. \& Schult., Eleocharis maculosa (Vahl) Roem. \& Schult. e Eleocharis sellowiana Kunth foram testados para atividade moluscicida, contra caramujos adultos e desovas, e toxicidade (ensaio de letalidade com Artemia salina). O extrato hexânico de Eleocharis acutangula (parte subterrânea fresca) foi ativo contra Artemia salina $\left(\mathrm{CL}_{50}=476,00 \mu \mathrm{g} / \mathrm{mL}\right)$, enquanto os demais extratos apresentaram $\mathrm{CL}_{50}$ $>10^{3} \mu \mathrm{g} / \mathrm{mL}$, sugerindo baixa toxicidade. O extrato hidro-etanólico de Eleocharis sellowiana (parte subterrânea fresca) foi ativo contra desovas de Biomphalaria glabrata $\left(\mathrm{CL}_{50}=24,27 \mu \mathrm{g} / \mathrm{mL}\right.$ ) mas inativo contra indivíduos adultos. Os demais extratos testados não apresentaram atividade moluscicida.
\end{abstract}

Unitermos: Cyperaceae, Eleocharis, atividade moluscicida, atividade ovicida, Biomphalaria glabrata, Artemia salina.

\begin{abstract}
Toxic evaluation of four species of the genus Eleocharis (Cyperaceae) in Artemia salina and Biomphalaria glabrata". The genus Eleocharis R. Br. comprises about 200 species, occurring in wet environments like swamps, lakes and river margins. In the search for new molluscicides, extracts from Eleocharis acutangula (Roxb.) Schult., Eleocharis interstincta (Vahl) Roem. \& Schult., Eleocharis maculosa (Vahl) Roem. \& Schult. and Eleocharis sellowiana Kunth were tested for molluscicidal activity (spawns and adult snails) and toxicity (Brine Shrimp Lethality - BSL - bioassay). The hexane extract of Eleocharis acutangula (fresh subterraneous parts) was active in the BSL bioassay $\left(\mathrm{LC}_{50}=476 \mu \mathrm{g} / \mathrm{mL}\right.$ ), while the other extracts showed $\mathrm{LC}_{50}>10^{3}$ $\mu \mathrm{g} / \mathrm{mL}$, suggesting they have low toxicity. The aqueous ethanol extract of Eleocharis sellowiana (fresh subterraneous parts) was active against Biomphalaria glabrata spawns $\left(\mathrm{LC}_{50}=24.27 \mu \mathrm{g} / \mathrm{mL}\right.$ ) but it was not lethal to adult snails. No other plant extract tested in this study showed molluscicidal activity.
\end{abstract}

Keywords: Cyperaceae, Eleocharis, molluscicidal activity, ovicidal activity, Biomphalaria glabrata, Artemia salina.

\section{INTRODUÇÃO}

Esquistossomose, causada pelo trematóide Schistosoma mansoni, é uma importante doença endêmica no Brasil e em muito outros países tropicais. O ciclo de vida desse parasita envolve um hospedeiro intermediário representado no Brasil por caramujos do gênero Biomphalaria (Alves et al., 2000), sendo o caramujo B. glabrata o principal vetor nas Américas do Sul e Central. De acordo com Paraense e Correa (1963), B. glabrata é considerado um agente transmissor modelo, provavelmente por causa de adaptações fisiológicas entre o molusco e a cepa local do parasita. Mais ainda, experimentos realizados por Zanotti-Magalhães et al. (1997) com espécimes coletados em Belo Horizonte (MG) confirmaram o alto potencial como vetor atribuído a B. glabrata.

Para o controle da esquistossomose, além do tratamento dos pacientes infectados, é muito importante que as populações de caramujos sejam controladas como uma forma de redução do risco de transmissão da doença. A niclosamida é o moluscicida comercialmente disponível recomendado pela Organização Mundial da Saúde (OMS) e provou ser mais efetivo e menos prejudicial ao meio ambiente e à saúde humana do que outros moluscicidas inorgânicos ou sintéticos, porém o alto custo de sua 
aplicação em áreas extensas torna proibitivo o seu uso na maioria dos países em desenvolvimento. Desta forma, a busca por moluscicidas naturais ganhou um novo destaque visando a obtenção de um produto alternativo mais barato, biodegradável, seguro e disponível localmente para controle das populações de caramujos (Clark et al., 1997; Oliveira-Filho; Paumgartten, 2000). Uma importante faceta da atividade de um eficiente moluscicida é sua capacidade de matar as desovas dos caramujos (Tang et al., 1995).

A fim de estabelecer a toxicidade de novos produtos naturais, muitos ensaios podem ser utilizados, como o ensaio de letalidade com o microcrustáceo Artemia salina, que foi desenvolvido para detectar compostos bioativos em extratos vegetais (Meyer et al., 1982; Nick et al., 1995), mas que também pode ser utilizado para expressar a toxicidade de um extrato com atividade moluscicida contra organismos não-alvos, como peixes e pequenos crustáceos (Oliveira-Filho; Paumgartten, 2000; Lima et al., 2002).

O gênero Eleocharis R. Br. (Cyperaceae, Cyperoideae, Scirpeae) abrange cerca de 200 espécies, que ocorrem em ambientes úmidos como pântanos e margens de lagos e rios. A parte aérea compreende um caule simples não ramificado que termina em uma inflorescência formada por inúmeras flores, enquanto um caule modificado, denominado rizoma ou estolão (dependendo da forma) e as raízes formam a parte subterrânea. E. sellowiana Kunth ocorre no Mexico, Colombia, Paraguai e Brasil, enquanto E. acutangula (Roxb.) Schult. ocorre na Ásia, Austrália, África, Índia, Japão e Américas do Sul e Central. Ambas as espécies são amplamente distribuídas pelo estado de São Paulo. Já E. maculosa (Vahl) Roem. \& Schult. e E. interstincta (Vahl) Roem. \& Schult. ocorrem na Índia e Américas do Sul e Central e são encontradas em apenas algumas áreas do estado de São Paulo (Faria, 1998). Destas quatro espécies, apenas E. sellowiana e E. acutangula já foram objeto de estudos fitoquímicos, resultando no isolamento de lupa-20(29)-eno-3 $\beta, 16 \beta$-diol, uma mistura de campesterol, stigmasterol e sitosterol, neohopa13(18)-eno-3 $\alpha$-ol, estigmasta-22-eno-3 $\beta, 6 \beta, 9 \alpha$-triol, ácido betulínico, estigmasta-4-eno-6 $\beta$-ol-3-ona, ferna9(11)-eno-3 $\alpha$-ol, $\quad 3 \alpha$-hidroxi-13 $\alpha, 17 \alpha, 21 \beta$-hopano15,19-diona e hexadecanoato de $E$-fitila além de dezoito ésteres de ácidos graxos, sete alcanos de cadeia longa, diidroactinidiolídeo, fitona, neofitadieno, curcumeno, $\alpha$-muuroleno e $\gamma$-cadineno (Ruiz, 2003, Amaral et al., 2004).

Neste contexto, os extratos de algumas espécies de Eleocharis foram submetidos aos ensaios com larvas de Artemia salina e com caramujos e desovas de $B$. glabrata, visando novas alternativas para o controle da esquistossomose.

\section{MATERIAL E MÉTODOS}

\section{Material Vegetal}

Amostras de todas as espécies foram coletadas na região de Campinas (SP, Brasil) e foram identificados por A. D. Faria e M. C. E. Amaral. Exsicata das espécies: E. sellowiana: A.D. Faria et al. 1000, UEC 103089; E. acutangula: A.D. Faria et al. 1001, UEC 103088; E. interstincta: L.Y.S. Aona, A.D. Faria \& G.Jacobucci 95/12, UEC 94218; E. maculosa: L.Y.S. Aona, A.D. Faria et al. 97/213, UEC 93701, estão depositados no Herbário do Departamento de Botânica, do Instituto de Biologia da Universidade Estadual de Campinas (UEC).

\section{Extração}

Plantas recentemente coletadas foram separadas em partes aéreas e subterrâneas. Extratos de ambas as partes frescas foram obtidos por maceração com etanol (5 x 1L, 24h de maceração cada) e após redução do volume (cerca de $200 \mathrm{~mL}$ ) e adição de água (200 mL), o extrato hidroetanólico bruto foi submetido a partição em funil de separação com hexano (4 x $200 \mathrm{~mL})$, acetato de etila (4 x $200 \mathrm{~mL})$ e $\mathrm{CHCl}_{3}(4 \times 200 \mathrm{~mL})$ sendo que a fração hidroetanólica final também foi concentrada. Partes aéreas moídas e secas foram extraídas em aparelho Soxhlet com hexano, $\mathrm{CH}_{2} \mathrm{Cl}_{2}$ e $\mathrm{MeOH}$ (4L cada) e os extratos resultantes foram concentrados. As quantidades empregadas de material vegetal, bem como as quantidades obtidas de extrato encontram-se resumidas na Tabela 1.

\section{Ensaio de letalidade com Artemia salina}

O ensaio foi realizado segundo o procedimento descrito na literatura (Meyer et al., 1982; Mclaughlin et al., 1995) e as concentrações letais abaixo de $10^{3}$ $\mu \mathrm{g} / \mathrm{mL}$ indicavam uma potencial atividade tóxica. Resumidamente, cada extrato (20 mg) foi dissolvido em $2 \mathrm{~mL}$ de solvente apropriado e dessa solução, amostras de 5, 50 e $500 \mu \mathrm{L}$ foram transferidas, em triplicata, para os frascos de $5 \mathrm{~mL}$. Após a remoção total do solvente, uma solução salina $(0,38 \mathrm{~g} / \mathrm{L}, 5 \mathrm{~mL})$ foi adicionada em cada um dos frascos, resultando em concentrações finais de 10, 100 e $1000 \mu \mathrm{g} / \mathrm{mL}$, respectivamente. Larvas do tipo nauplii de $A$. salina (10 por frasco) foram adicionadas e após 24 h de contato, os sobreviventes foram contados. Os dados foram analisados através do método de Probit (Finney, 1962) e expressos como $\mathrm{CL}_{50}$ (Tabela 1).

\section{Moluscos}

Foram utilizados caramujos $B$. glabrata melanicos, com 10-15 mm de diâmetro, coletados de Belo Horizonte (MG) e mantidos em aquários com circulação contínua de água desclorada e à temperatura de $28{ }^{\circ} \mathrm{C}$, no Departamento de Parasitologia, Instituto de Biologia, Unicamp. 
Ensaio de atividade moluscicida contra indivíduos adultos

O ensaio foi realizado segundo o procedimento descrito na literatura (Magalhães et al., 2003) e as concentrações letais abaixo de $100 \mu \mathrm{g} / \mathrm{mL}$ indicavam uma potencial atividade moluscicida. Resumidamente, cinco caramujos B. glabrata de tamanho uniforme (diâmetro da concha entre 8-10 mm) foram colocados em contato com $30 \mathrm{~mL}$ de solução aquosa de concentração conhecida (25 e $250 \mu \mathrm{g} / \mathrm{mL}$ ) do extrato vegetal, enquanto grupos de controle foram mantidos em água desclorada. Após 24h, os caramujos foram lavados e mantidos em frascos com água limpa e desclorada e observou-se a ocorrência de

Tabela 1. Resultados dos ensaios de letalidade com A. salina e de atividade moluscicida (caramujos adultos e desovas)

\begin{tabular}{|c|c|c|c|c|c|}
\hline \multirow[t]{2}{*}{ Espécie } & \multirow[t]{2}{*}{ Parte $(g)$} & \multirow[t]{2}{*}{ Extrato (mg) } & \multirow{2}{*}{$\begin{array}{c}\text { Artemia salina } \\
\qquad(\mu \mathrm{g} / \mathrm{mL})\end{array}$} & \multicolumn{2}{|c|}{$\begin{array}{l}\text { Biomphalaria glabrata } \\
\qquad(\mu \mathrm{g} / \mathrm{mL})\end{array}$} \\
\hline & & & & Caramujos & Desovas \\
\hline \multirow[t]{6}{*}{ E. acutangula } & Aérea fresca & $\mathrm{H}(869,0)$ & - & NT & NT \\
\hline & $(187,4)$ & $\mathrm{AE}(1075,9)$ & - & NT & NT \\
\hline & & HE $(2023,7)$ & - & - & - \\
\hline & Subterrânea fresca & $\mathrm{H}(145,3)$ & 476,00 & NT & NT \\
\hline & $(111,9)$ & $\operatorname{AE}(125,6)$ & - & NT & NT \\
\hline & & $\operatorname{HE}(1267,5)$ & - & - & - \\
\hline \multirow[t]{3}{*}{ E. interstincta } & Aérea fresca & $\mathrm{H}(1779,6)$ & - & NT & NT \\
\hline & $(207,5)$ & $\mathrm{AE}(742,3)$ & - & NT & NT \\
\hline & & HE $(6590,0)$ & - & - & - \\
\hline \multirow[t]{6}{*}{ E. maculosa } & Aérea fresca & H $(907,8)$ & - & NT & NT \\
\hline & $(210,0)$ & $\mathrm{AE}(525,4)$ & - & NT & NT \\
\hline & & $\operatorname{HE}(3528,5)$ & - & - & - \\
\hline & Aérea seca & $\mathrm{H}(1485,4)$ & - & NT & NT \\
\hline & $(98,6)$ & $\mathrm{C}(605,0)$ & - & NT & NT \\
\hline & & $\mathrm{M}(8708,1)$ & - & - & - \\
\hline \multirow[t]{9}{*}{ E. sellowiana } & Aérea fresca & $\mathrm{H}(1283,4)$ & - & NT & NT \\
\hline & $(270,0)$ & $\mathrm{AE}(670,9)$ & - & NT & NT \\
\hline & & $\operatorname{HE}(3105,2)$ & - & - & - \\
\hline & Subterrânea fresca & $\mathrm{H}(358,4)$ & - & NT & NT \\
\hline & $(250,5)$ & C $(1303,4)$ & - & NT & NT \\
\hline & & HE $(2984,7)$ & - & - & 24,27 \\
\hline & Aérea seca & $\mathrm{H}(2249,3)$ & - & NT & NT \\
\hline & $(95,8)$ & $\mathrm{C}(211,2)$ & - & NT & NT \\
\hline & & $\mathrm{M}(2352,0)$ & - & - & - \\
\hline
\end{tabular}

H: hexano; AE: acetato de etila; HE: hidro-etanólico; C: clorofórmio; M: metanol; (-): inativo; NT: não testado. 
Tabela 2. Desenvolvimento comparativo de desovas de Biomphalaria glabrata na presença e na ausência de Eleocharis sellowiana no aquário

\begin{tabular}{lcccc}
\hline & \multicolumn{3}{c}{ Ovos viáveis } \\
\cline { 2 - 5 } & \multicolumn{2}{c}{ Desenvolvimento com E. sellowiana } & Desenvolvimento sem E. sellowiana \\
\cline { 2 - 5 } & Vivos & Mortos & Vivos & Mortos \\
\hline Início & 236 & 0 & 237 & 0 \\
Após 24 h & 236 & 0 & 237 & 0 \\
Após 3 dias & 236 & 0 & 235 & 2 \\
Após 7 dias & 236 & 0 & 232 & 5 \\
Após 15 dias & 225 & 11 & 230 & 7 \\
\hline
\end{tabular}

mortalidade depois de um período de 24h, utilizando-se como critério ausência de movimento. Os ensaios foram feitos em duplicata.

\section{Atividade moluscicida contra desovas (atividade ovicida)}

Este ensaio foi realizado segundo a metodologia descrita por Okazaki et al. (1996) com algumas modificações. Desovas de B. glabrata foram coletadas através de filmes plásticos colocados na superfície dos aquários dos caramujos. Para cada teste, seis desovas com 20 a 30 ovos, em estágio de blástula (24-h) foram colocadas em contado com $30 \mathrm{~mL}$ de solução aquosa do extrato vegetal (25 e $250 \mu \mathrm{g} / \mathrm{mL}$ ) enquanto grupos de controle foram colocados em água desclorada. Após um período de exposição de 24h, as desovas foram lavadas em água limpa e mantidas em placas de Petri com água desclorada, a $25{ }^{\circ} \mathrm{C}$ durante 15 dias, tempo médio para eclosão dos ovos. Cada desova foi observa em microscópio para verificar a viabilidade dos embriões antes do início do teste, após $24 \mathrm{~h}$ de contato e no terceiro, sétimo e décimo quinto dias para contagem dos embriões vivos. Os dados foram analisados através do método de Probit (Finney, 1962) e expressos como $\mathrm{CL}_{50}$ (Tabela 1).

\section{Ensaio in vivo}

Algumas mudas de E. sellowiana foram plantadas em um aquário onde algumas desovas (ca. 15 ovos cada) de $B$. glabrata foram colocadas e o desenvolvimento destas foi acompanhado durante 15 dias, tempo médio para eclosão dos ovos. O experimento foi realizado em duplicata e aquários apenas com as desovas foram utilizados como grupo controle (Tabela 2).

\section{RESULTADOS}

Todos os extratos obtidos foram testados contra
A. salina enquanto apenas os extratos metanólicos e hidro-etanólicos (solúveis em água) foram testados contra caramujos e desovas de B. glabrata. Todos os resultados estão resumidos nas Tabelas 1 e 2, mostrando não haver diferenças significativas entre o material seco ou fresco. Já os extratos das partes subterrâneas mostraram-se mais promissores do que os obtidos das partes aéreas, nas condições empregadas.

\section{DISCUSSÃO}

Esta é a primeira vez que extratos obtidos de espécies do genêro Eleocharis são submetidos à ensaios de atividade moluscicida e de toxicidade. Dentro da família Cyperaceae, foi encontrado apenas o relato de atividade moluscicida dos extratos de Cyperus luzulae contra caramujos adultos Biomphalaria glabrata (Marston et al., 1996).

Os resultados obtidos no ensaio com $A$. salina indicam uma baixa toxicidade dos extratos testados, uma vez que quase todos apresentaram $\mathrm{CL}_{50}>>10^{3}$ $\mu \mathrm{g} / \mathrm{mL}$, exceto o extrato hexânico de E. acutangula (parte subterrânea) que apresentou $\mathrm{CL}_{50}=476,00 \mu \mathrm{g} /$ mL (Tabela 1). A toxicidade com A. salina mostra boa correlação com atividades antitumoral, inseticida (Meyer et al., 1982; Mclaughlin et al., 1995) e anti-Trypanosoma cruzi (Alves et al., 2000) para substâncias com $\mathrm{CL}_{50}<$ $10^{3} \mu \mathrm{g} / \mathrm{mL}$. Por outro lado, uma baixa toxicidade pode ser considerada uma característica interessante para utilização de extratos vegetais em ambientes naturais para controle da população de caramujos. É relevante observar que a niclosamida mostrou-se tóxica para espécies aquáticas como crustáceos do zooplancton (OliveiraFilho; Paumgartten, 2000).

Assim, os extratos metanólicos e hidroetanólicos foram testados para as atividades moluscicida e ovicida por causa de sua solubilidade em água e sua baixa toxicidade. Contra caramujos adultos nenhum dos extratos mostrou-se ativo (Tabela 1) enquanto que o extrato hidro-

\begin{tabular}{r|r}
\hline Rev. Bras. Farmacogn. & 101 \\
Braz J. Pharmacogn. & 101 15(2):abr/jun. 2005
\end{tabular}


etanólico de E. sellowiana (parte subterrânea) foi ativo contra desovas de B. glabrata $\left(\mathrm{CL}_{50}=24,27 \mu \mathrm{g} / \mathrm{mL}\right)$. Foi interessante observar que as desovas apresentavamse com aspecto normal até a segunda observação, isto é, grande parte dos embriões morria entre o terceiro e o sétimo dia após a exposição ao extrato. Por causa dessa atividade, propôs-se observar se a planta poderia controlar o desenvolvimento de B. glabrata sob condições naturais, uma vez que ambos podem compartilhar o mesmo ambiente úmido. Os resultados obtidos no experimento in vivo, entretanto, não confirmaram essa hipótese uma vez que não houve diferenças significativas na mortalidade de desovas entre os aquários com e sem E. sellowiana (Tabela 2).

Dessa forma, a atividade ovicida observada para o extrato hidro-etanólico de E. sellowiana (parte subterrânea), aliada à baixa toxicidade e a facilidade de preparo e de solubilidade em água desse extrato, sugere que o mesmo pode vir a ser empregado no controle de populações de caramujos B. glabrata, resaltando ainda que $E$. sellowiana ocorre naturalmente em quase todo o Brasil e pode ser facilmente cultivada. Estas características são algumas daquelas apresentadas por Clark et al (1997) como importantes na escolha de um moluscicida ideal.

\section{AGRADECIMENTOS}

Os autores agradecem à FAPESP pelo suporte financeiro e pelas bolsas de estudo concedidas a Ana Lúcia T. G. Ruiz, Aparecida D. de Faria and Deborah R. Serrano. A Profa. Dra. Maria do Carmo E. do Amaral também agradece ao CNPq por uma bolsa de pesquisa. Este trabalho é parte da tese de doutorado de Ana Lúcia T. G. Ruiz.

\section{REFERÊNCIAS}

Alves TMD, Silva AF, Brandão M, Grandi TSM, Smânia EFA, Smânia A, Zani CL 2000. Biological screening of Brazilian medicinal plants. Mem Inst Oswaldo Cruz 95: 367-373.

Amaral MCE, Faria AD, Magalhães AF, Magalhães EG, Ruiz ALTG 2004. Steroids and triterpenes from Eleocharis acutangula and Eleocharis sellowiana (Cyperaceae). Phytochem Anal 15: 125-129.

Clark TE, Appleton CC, Drewes SE 1997. A semi-quantitative approach to the selection of appropriate candidate plant molluscicides - A South African application. $J$ Ethnopharmacol 56: 1-13.

Faria AD 1998. O gênero Eleocharis R. Br. (Cyperaceae) no Estado de São Paulo. Campinas, 200p. Dissertação de Mestrado - Instituto de Biologia, Universidade Estadual de Campinas.

Finney DJ 1962. Probit Analysis. Cambridge, Cambridge University Press.

Lima NMF, Santos AF, Profírio Z, Goulart MOF, Sant'ana AEG 2002. Toxicity of lapachol and their potassium solts against Biomphalaria glabrata, Schistosoma mansoni cercariae, Artemia salina and Tilapia nilotica. Acta
Trop 83: 43-47.

Magalhães AF, Tozzi AMGA, Santos CC, Serrano DR, ZanottiMagalhães EM, Magalhães EG, Magalhães LA 2003. Saponins from Swartzia langsdorffii: biological activities. Mem Inst Oswaldo Cruz 98: 713-718.

Marston A, Dudan G, Gupta MP, Solis PN, Correa MD, Hostettmann K 1996. Screening of Panamanian plants for molluscicidal activity. Int J Pharm 34: 15-18.

Mclaughlin JL, Saizarbitori T-C, Anderson JE 1995. Tres bioensayos simples para quimicos de productos naturales. Rev Soc Venez Quim 18: 13-18.

Meyer BN, Ferrigni NR, Putnam JE, Jacobsen LB, Nichols DE, Mclaughlin JL 1982. Brine shrimp, a convenient general bioassy for active-plant constituents. Planta Med 45: 31-34.

Nick A, Rali T, Sticher O 1995. Biologial screening of traditional medicinal plants from Papua New Guinea. J Ethnopharmacol 49: 147-156.

Okazaki K, Andrade Junior HF, Kawano T 1996. Effect of ${ }^{60} \mathrm{Co}$ gamma radiation on Biomphalariaglabrata (Mollusca, Gastropoda) embryos: mortality, malformation and hatching. Braz J Med Biol Res 29: 1057-1067.

Oliveira-Filho EC, Paumgartten FJR 2000. Toxicity of Euphorbia milii latex and niclosamide to snails and nontarget aquatic species. Ecotox Environ Safe 46: 342-350.

Paraense WL, Correa LR 1963. Variation in susceptibility of populations of Australorbis glabratus to strain of Schistosoma mansoni. Rev Inst Med Trop 5: 15-22.

Ruiz ALTG 2003. Estudo fitoquímico de algumas espécies do gênero Eleocharis R. Br. (Cyperaceae): isolamento, elucidação estrutural e atividade biológica. Campinas, 200p. Tese de Doutorado - Instituto de Química, Universidade Estadual de Campinas.

Tang SH, Whitfield PJ, Perrett S 1995. Activity of the molluscicidal plant Milletia thonnigii (Leguminosae) toward Biomphalaria glabrata eggs. J Parasitol 81: 833-835.

Zanotti-Magalhães EM, Magalhães LA, Carvalho JF 1997. Relação entre a patogenicidade do Schistosoma mansoni em camundongos e a susceptibilidade do molusco vetor. IV - Infecciosidade dos miracídeos. Rev Saude Publ 31: 448-494. 